\title{
Denying the wolf access to sheep's clothing
}

\author{
Theo Heller and Jay H. Hoofnagle \\ Liver Diseases Section, National Institute of Diabetes and Digestive \\ and Kidney Diseases, National Institutes of Health, Bethesda, Maryland, USA
}

\begin{abstract}
Hepatitis delta virus (HDV) is a cause of acute and chronic liver disease for which no effective therapy is currently available. Previous research has demonstrated that prenylation of the large HDV antigen is essential for viral assembly. A new report (see the related article beginning on page 407) describes a novel small animal model for HDV replication and demonstrates that prenylation inhibitors are highly effective at clearing viremia and thus have potential relevance for the treatment of chronic delta hepatitis.
\end{abstract}

J. Clin. Invest. 112:319-321 (2003). doi:10.1172/JCI200319417.

Delta hepatitis is the least frequent but most severe form of viral hepatitis. Acute infection with hepatitis delta virus (HDV) is often severe and has a fatality rate of at least $5 \%$, even in children. Chronic delta hepatitis leads to cirrhosis in at least two thirds of persons and can present as endstage liver disease in early adulthood (1). Hepatitis D also has the distinction of being the form of viral hepatitis that is most resistant to current antiviral therapies (2).

\section{Virology of HDV}

The unique clinical features of delta hepatitis are matched by the unique qualities of its viral agent. HDV is the smallest known human pathogen and uses a unique replicative strategy (3). The HDV genome is merely $1.7 \mathrm{~kb}$ in length and consists of a singlestranded circular molecule of RNA of negative polarity that is held in a rigid rod-like form by extensive intramole-

\footnotetext{
Address correspondence to: Jay $\mathrm{H}$.

Hoofnagle, Liver Diseases Research Branch, National Institute of Diabetes and Digestive and Kidney Diseases, National Institutes of Health, Building 31, Room 9A27, Bethesda, Maryland 20892, USA.

Phone: (301) 496-1333; Fax: (301) 480-7926;

E-mail: hoofnaglej@extra.niddk.nih.gov.

Conflict of interest: The authors have

declared that no conflict of interest exists.

Nonstandard abbreviations used: hepatitis delta virus (HDV); hepatitis B virus (HBV); hepatitis B surface antigen (HBsAg); small HDV antigen (S); large HDV antigen (L).
}

cular base pairing (4). The antigenome of HDV RNA has a single open reading frame that encodes hepatitis delta antigen. Importantly, HDV requires the presence of another (helper) virus for productive replication and spread (5). The helper virus is the hepatitis B virus (HBV), a very significant human pathogen in its own right. Being linked to HBV and only occurring in persons who have hepatitis B surface antigen (HBsAg) in serum, HDV is spread in the same ways as hepatitis $B$, through parenteral or sexual exposure to blood or body fluids. Up to $5 \%$ of the world's population is infected with HBV, and probably $5 \%$ of these have HDV superinfection (6). Delta hepatitis has a worldwide distribution, but areas of dramatically high endemicity associated with severe clinical outcomes have been identified in the Amazon basin, Central Africa, and parts of Eastern Europe. Prevention of hepatitis B will also prevent hepatitis D. In a triumph of public health, increasing vaccination rates for hepatitis $\mathrm{B}$ are contributing to the decline in HDV incidence. Nevertheless, there are still millions of persons with chronic HDV infection, and until hepatitis B is fully eradicated, new cases of delta hepatitis will occur.

HDV circulates in serum as a 35- to 40-nm particle containing viral RNA and HDV antigen, encapsidated by
$\operatorname{HBsAg}(7,8)$. The envelope protein of HBV allows for the specific binding and uptake of virions into hepatocytes. Inside the cell, the viral genome is released and replicated by a doublerolling circle mechanism (4). Replication of HDV, like that of other viruses, requires multiple enzymatic activities. Although many viruses encode the majority of their replicative and processing enzymes, HDV apparently does not. The sole enzymatic activity HDV possesses is a ribozyme (similar to that in plant satellite viruses) that autocleaves the circular RNA, producing a linear molecule (9). Other enzyme activities are apparently provided by the host cell.

\section{Editing of HDV RNA and prenylation of the large HDV antigen}

HDV antigen is produced in infected cells in two sizes, small (S) and large (L), the $\mathrm{L}$ form having an additional 19-20 amino acids on its carboxyl terminus (10). It was initially unclear how a single open reading frame encoded two different-sized polypeptides. In a series of elegant studies, Casey and coworkers demonstrated that HDV RNA is edited by a cellular enzyme (double-stranded RNA adenosine deaminase) that change $\mathrm{A}$ to I on the antigenomic (+) strand, which with replication causes a change in the UAG stop codon to UGG, which encodes tryptophan (Figure 1). Without the initial stop codon, a larger delta antigen is created that is terminated 19-20 codons downstream by a second stop codon. Virtually all strains of HDV use this editing mechanism.

In complementary studies, Glenn and coworkers demonstrated that the L HDV antigen had a prominent isoprenylation site near the added carboxyl terminus (11). Addition of a farnesyl molecule to the site creates a lipophilic area, allowing for membrane association and virion assembly. The association of HBsAg and prenylated L HDV antigen thus promotes packaging of the virus and export. These two lines of research pointed to how HDV replication 


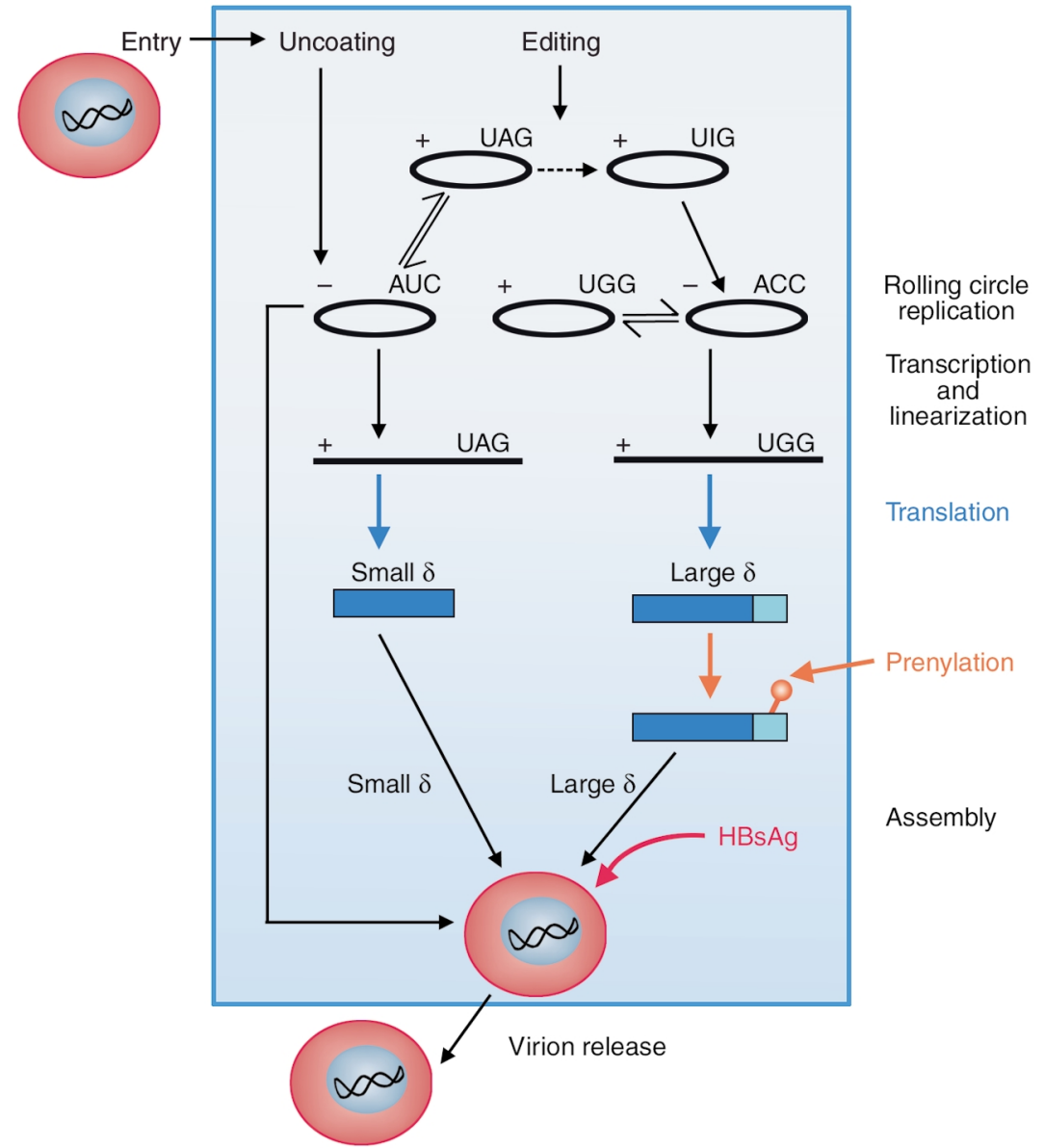

Figure 1

Replication of HDV. Viral particles consist of HDV RNA (black lines) and HDV antigen (blue) encapsidated by HBsAg (red). The HBsAg allows for binding and uptake of the virion into hepatocytes. Inside the cell, the RNA genome (negative polarity) is released and undergoes replication by the double-rolling circle pattern (double arrows). The antigenomic RNA (positive polarity) serves as the template for production of more genomic RNA but can also be edited by intracellular host dsRNA adenosine deaminase, changing $A$ to I so that with production of genomic RNA, C rather than $U$ will be transcribed (right side of the figure). The editing changes the UAG stop codon on the positive strand to a UGG, which is read as tryptophan, resulting in a protein with an additional 19 amino acids, the large delta antigen (large $\delta$ ) that inhibits replication. Small delta antigen (small $\delta$ ), the product of the unedited transcript (left side of the figure), binds to HDV RNA and promotes replication. Large delta antigen has a prenylation site that creates a hydrophobic molecule that binds both to HDV RNA and to HBsAg, leading to viral assembly and release. Thus, inhibition of prenylation will interfere with viral assembly and release but should affect replication minimally, if at all.

depends on cellular enzymes that the virus redirects toward production of viral antigen and virions. HDV also clearly uses other cellular enzymes, not all of which are well defined but perhaps include a helicase to unwind the intramolecular base pairing of the HDV RNA circular genome and a polymerase for production of viral RNA. These findings are important because knowledge of the viral life cycle can point to targets for antiviral prenylation of the L HDAg and show how this knowledge can be used to develop therapies for delta hepatitis. Since prenylation is critical for virion formation, prenylation inhibitors might be effective in blocking viral replication. The hurdle for demonstrating activity of prenylation inhibitors (many of which were developed as anticancer agents) (13) was the lack of availability of a practical model for studying the viral life cycle. To this end, the authors developed a murine model using hydrodynamic transfection of HDV cDNA into an established HBV transgenic mouse. They reasoned that if HBsAg was provided, introduction of HDV RNA might result in HDV viremia. This was indeed the case. The authors then showed that viremia was blocked by prenylation inhibition.

At present, therapy of delta hepatitis is difficult and unsatisfactory. The nucleoside analogues that block HBV replication have little or no effect against HDV replication or the disease that it causes. IFN- $\alpha$ inhibits HDV replication, but therapy usually requires high doses given for a prolonged period, which are often poorly tolerated (2).

The work by Bordier et al. (12) can be viewed as a new phase of therapeutics for HDV. In the typical first stage, known and established antiviral drugs are taken off the shelf and tried. Such was the case with studies of IFN- $\alpha$ and the nucleoside analogue lamivudine for $\operatorname{HDV}(2,14)$. In the second stage, fundamental knowledge about the virus is applied, and drugs are used on the basis of such knowledge. With prenylation inhibitors, hepatitis $\mathrm{D}$ may enter the second stage of drug discovery, an excellent example of translational research. For the third phase, drugs are specifically designed and synthesized on the basis of the structure and life cycle of the virus. Delta hepatitis may be the least common form of viral hepatitis, but in many ways it is highly deserving of focused drug discovery.

\footnotetext{
1. Rizzetto, M., et al. 1983. Chronic hepatitis in carriers of hepatitis B surface antigen, with intrahepatic expression of the delta antigen. An active and progressive disease unresponsive to immunosuppressive treatment. Ann. Intern. Med. 98:437-441.
} 
2. Hadziyannis, S.J. 1991. Use of alpha-interferon in the treatment of chronic delta hepatitis. J. Hepatol. 13(Suppl. 1):S21-S26.

3. Wang, K.S., et al. 1986. Structure, sequence and expression of the hepatitis delta $(\delta)$ viral genome. Nature. 323:508-514.

4. Branch, A.D., and Robertson, H.D. 1984. A replication cycle for viroids and other small infectious RNA's. Science. 223:450-455.

5. Rizzetto, M., et al. 1980. Transmission of the hepatitis B virus-associated delta antigen to chimpanzees. J. Infect. Dis. 141:590-602.

6. Gaeta, G.B., Stornaiuolo, G., and Precone, D.F. 2001. Type B and D viral hepatitis: epidemio- logical changes in Southern Europe. Forum (Genova). 11:126-133.

7. He, L.F., et al. 1993. The size of the hepatitis delta agent. J. Med. Virol. 27:31-33.

8. Ryu, W.S., Netter, H.J., Bayer, M., and Taylor, J. 1993. Ribonucleoprotein complexes of hepatitis delta virus. J. Virol. 67:3281-3287.

9. Sharmeen, L., Kuo, M.Y., Dinter-Gottlieb, G., and Taylor, J. 1988. Antigenomic RNA of human hepatitis delta virus can undergo selfcleavage. J. Virol. 62:2674-2679.

10. Casey, J.L., Bergmann, K.F., Brown, T.L., and Gerin, J.L. 1992. Structural requirements for RNA editing in hepatitis delta virus: evidence for a uridine-to-cytidine editing mechanism. Proc Natl. Acad. Sci. U. S. A. 89:7149-7153.

11. Glenn, J.S., Watson, J.A., Havel, C.M., and White, J.M. 1992. Identification of a prenylation site in delta virus large antigen. Science. 256:1331-1333.

12. Bordier, B.B., et al. 2003. In vivo antiviral efficacy of prenylation inhibitors against hepatitis delta virus. J. Clin. Invest. 112:407-414. doi:10.1172/JCI200317704.

13. Schafer, W.R., and Rine, J. 1992. Protein prenylation: genes, enzymes, targets, and functions. Annu. Rev. Genet. 26:209-237.

14. Lau, D.T., et al. 1999. Lamivudine for chronic delta hepatitis. Hepatology. 30:546-549.

\title{
Amyloid $\beta$ and Alzheimer disease therapeutics: the devil may be in the details
}

\section{John R. Cirrito ${ }^{1}$ and David M. Holtzman ${ }^{1,2,3}$}

${ }^{1}$ Department of Neurology,

${ }^{2}$ Department of Molecular Biology and Pharmacology, and

${ }^{3}$ Center for the Study of Nervous System Injury, Washington University

School of Medicine, St. Louis, Missouri, USA

\begin{abstract}
Alzheimer disease $(\mathrm{AD})$ is characterized by the progressive accumulation of amyloid $\beta$ protein $(A \beta)$ in areas of the brain serving cognitive functions such as memory and language. The first of two separate reports (see the related articles beginning on pages 415 and 440) reveals that intrinsic $T$ cell reactivity to the self-antigen $A \beta$ exists in many humans and increases with age. This finding has implications for the design of $A \beta$ vaccines. The second report demonstrates that a number of FDA- approved nonsteroidal anti-inflammatory drugs are capable of lowering $A \beta$ levels in mice. The work suggests that further testing of the therapeutic utility of these types of compounds for the potential treatment of $\mathrm{AD}$ is warranted.
\end{abstract}

J. Clin. Invest. 112:321-323 (2003). doi:10.1172/JCI200319420.

Alzheimer disease $(\mathrm{AD})$ has received a lot of recent attention, particularly in areas related to novel treatments. Recently, the potential therapeutic usefulness of the immune system has become apparent, leading to the question of whether it can be used to directly or indirectly influence ADrelated pathology in beneficial ways.

\footnotetext{
Address correspondence to: David M. Holtzman, Washington University School of Medicine, Department of Neurology, 660 South Euclid Avenue, Box 8111, St. Louis, Missouri 63110, USA. Phone: (314) 747-0286; Fax: (314) 362-2826; E-mail: holtzman@neuro.wustl.edu. Conflict of interest: The authors have declared that no conflict of interest exists. Nonstandard abbreviations used: Alzheimer disease (AD); amyloid $\beta$ protein $(\mathrm{A} \beta)$; amyloid precursor protein (APP); cerebral amyloid angiopathy (CAA).
}

Active immunization with amyloid $\beta$ $(A \beta)$ peptides takes advantage of the immune system to generate antibodies that can somehow decrease $A \beta$ related pathology in mouse models of AD (1). Similarly, passive immunization involves direct administration of anti-A $\beta$ antibodies, bypassing the need for an active immune response $(2,3)$. Since genetic, pathologic, and animal studies suggest that the buildup of $A \beta$ in the brain leads directly or indirectly to cell dysfunction, cell death, and cognitive impairment, increased generation of anti-A $\beta$ antibodies has the potential to prevent or treat $\mathrm{AD}$ by decreasing amyloid burden and its consequences in the brain. Though the first clinical trials for $A \beta$ vaccination were halted due to CNS inflammation in a small sub- set of subjects, active and passive immunization strategies remain a viable potential therapy worth continued exploration. If positive effects can be seen in future trials, it will be important to minimize unwanted toxicity. In this issue of the JCI, Monsonego and colleagues (4) further characterize the innate immune response to $A \beta$ in humans, thus revealing important details about how the elderly body reacts to $A \beta$, and opening new avenues to modify existing vaccination protocols. Also in this issue, Eriksen and colleagues (5) studied traditional NSAIDs that appear to have a nontraditional, COX-independent effect on decreasing $A \beta 42$ production. While these drugs are often used to treat inflammation, they appear to have a novel effect on amyloid precursor protein (APP) cleavage, which is only now becoming apparent and which may be useful in the future as a therapeutic.

\section{A $\beta$-reactive T cells increase with age}

Monsonego et al. (4) found that some healthy, elderly individuals, as well as individuals with $\mathrm{AD}$, contain elevated baseline levels of $A \beta$-reactive $T$ cells. While the general trend is toward a diminished immune response with aging, this demonstrates a selective increase in $\mathrm{A} \beta$-reactive $\mathrm{T}$ cells in older individuals with and without dementia. The reason for this selective expansion of $A \beta$-reactive $T$ cells in elderly individuals remains unclear. It is often presumed that cognitively normal middle-aged and elderly individuals are similar in that they lack AD pathology; however, $A \beta$ deposition in plaques appears to begin about $10-20$ years prior to the onset of even the earliest symptoms sugges- 